\title{
AS AÇÕES PEDAGÓGICAS PARA A INTERVENÇÃO DO TREINADOR DE SURF
}

\author{
THE PEDAGOGICAL ACTIONS FOR THE SURF COACH INTERVENTION
LAS ACCIONES PEDAGÓGICAS PARA LA INTERVENCIÓN DEL ENTRENADOR DE SURF
Vinicius Zeilmann Brasil ${ }^{\star}$, Valmor Ramos ${ }^{\star *}$, Jeferson Rodrigues de Souza*, Thais Emanuelli da Silva de Barros*, Juarez Vieira do Nascimento*

\begin{abstract}
Palavras-chave Aprendizagem. Esportes. Desenvolvimento pessoal.

Surf.

Resumo: 0 objetivo deste estudo foi analisar as ações pedagógicas para o ensino do surf a partir do entendimento de formadores de treinadores de surf. Foram entrevistados cinco formadores experientes, e as informações analisadas por meio dos procedimentos de estudo de casos. Os resultados indicam que as ações pedagógicas para o treinador de surf devem estar voltadas para a adaptação de sua intervenção às constantes alterações do ambiente de prática e para atender as expectativas do aprendiz. Concluiuse que as ações do treinador de surf devem estar pautadas na gestão do risco oferecido pela prática do surf, bem como na garantia de níveis adequados de risco, favorecendo a aprendizagem da modalidade.
\end{abstract}

\section{Keywords}

Learning.

Sports.

Staff development. Surf.

Palabras clave Aprendizage. Deportes.

Desarollo personal. Surf.

Abstract: The aim of this study was to analyze the pedagogical actions on surf teaching based on the understanding of surfing coaches' developers. Five experienced developers were interviewed and the information obtained was analyzed through case study procedures. The results indicate that the pedagogical activities for the surfing coach should be aimed at adapting its intervention to an ever-changing practice environment and to meet the learner's expectations. It was concluded that the surf coach's actions must be guided by management of the risks offered by surfing as well as by ensuring appropriate levels of risk that favor learning the sport.

Resumen: El objetivo de este estudio fue analizar las acciones pedagógicas para la enseñanza del surf con base en la comprensión de los formadores de entrenador de surf. Fueron entrevistados cinco formadores con experiencia y las informaciones analizadas por medio de procedimientos de estudio de caso. Los resultados indican que las acciones pedagógicas del entrenador de surf deben estar dirigidas a la adaptación de su intervención a los cambios del ambiente de práctica y para cumplir con las expectativas del alumno. Se concluyó que las acciones del entrenador surf deben estar guiada por la gestión de riesgos que ofrece la práctica de surf, así como para asegurar niveles adecuados de riesgo que favorecen el aprendizaje de la modalidad.
* Universidade Federal de Santa Catarina (UFSC). Florianópolis, SC, Brasil. E-mail: vzbrasil@ hotmail.com

** Universidade do Estado de Santa Catarina (UDESC). Florianópolis, SC, Brasil. E-mail: valmor.ramos@udesc.br

Recebido em: 27-07-2015 Aprovado em: 08-12-2015 (c) (1) (8) Licence 


\section{INTRODUÇÃO}

A necessidade de se compreender a prática pedagógica do treinador e contribuir para a formação dos próprios treinadores (DUFFY et al., 2011) tem sido um dos principais impulsionadores das pesquisas acerca da intervenção profissional do treinador esportivo (GILBERT; RANGEON, 2011) e, mais recentemente, do treinador de esportes de aventura (BERRY; LOMAX; HODGSON, 2015). Segundo Barnson (2014), esses estudos buscam um melhor entendimento dos conhecimentos utilizados por treinadores eficazes, especificamente, nas perspectivas sociocultural (CASSIDY, 2010), pedagógica (JONES; WALLACE, 2005), de eficácia (CÔTÉ; GILBERT, 2009) e holística (LYLE, 2002, 2007).

Essas pesquisas têm sido realizadas sob a influência da psicologia cognitiva, seguindo o paradigma investigativo denominado "Pensamento e Ação do Professor", centrado nos processos que ocorrem na mente dos professores a respeito de sua atividade profissional (CLARK; PETERSON, 1986; JANUÁRIO, 1996). No âmbito dos estudos sobre o treinador esportivo, esse paradigma de pesquisa é uma possibilidade de identificar características específicas do conhecimento profissional do treinador, seja a partir da descrição de modelos de atuação que representem uma estrutura conceitual, isto é, os processos envolvidos no treino esportivo, seja pela a maneira como essas estruturas interagem na prática (LYLE, 2002; CUSHION; ARMOUR; JONES, 2006).

Os Modelos do Treinador (Models of Coaching) correspondem a constructos que fornecem uma representação conceitual dos aspectos envolvidos na intervenção pedagógica do treinador, mediante dados empíricos obtidos em pesquisas com treinadores experts (CUSHION; ARMOUR; JONES, 2006). Na literatura especializada, as propostas são denominadas modelos, a exemplo do Coaching Model (CÔTÉ et al., 1995), do Coaching Schematic (ABRAHAM; COLLINS; MARTINDALE, 2006) e, atualmente, do Authentic Coaching Model (BARNSON, 2014), do Model of Olympic Podium Performance (DIN; PASKEVICH, 2013) e do Effective Coaching Model (BENNIE; O'CONNOR, 2011). Particularmente, o Coaching Model (CM), de Côté et al. (1995), tem inspirado muitos estudos sobre a prática dos treinadores (DIN; PASKEVICH, 2013), destacadamente nos esportes coletivos (BLOOM; SALMELA, 2000; DURAND-BUSH; SALMELA 2002; GILBERT; TRUDEL, 2000).

A proposta do Adventure Sports Coach Model (ASCM), de Collins e Collins (2012), embora não tenha sido elaborada a partir de dados empíricos de treinadores ligados a esses esportes, tem contribuído para estabelecer conceitos a respeito da intervenção profissional nessa área, esclarecendo as funções, os conhecimentos e as ações do Treinador de Esportes de Aventura (TEA). A proposta, portanto, foi formulada com base nos estudos sobre os motivos para a prática de esportes de aventura, a educação ao ar livre, a liderança no esporte, a tomada de decisão e a gestão do risco.

Não obstante o reconhecimento da contribuição dos modelos conceituais para a compreensão da prática do treinador (DIN; PASKEVICH, 2013), ainda se questiona o potencial limitado dessas propostas em representar a complexidade situacional inerente à intervenção profissional (BOWES; JONES, 2006; JONES; WALLACE, 2005), o que, no caso do surf, acentua-se devido à imprevisibilidade do ambiente de prática. Além disso, o desenvolvimento científico obtido nos estudos sobre a intervenção pedagógica do treinador de modalidades convencionais não parece ter implicações claras no campo da intervenção profissional dos 
esportes de aventura (BERRY; LOMAX; HODGSON, 2015; COLLINS; COLLINS, 2012), inclusive, na prática pedagógica do treinador de surf.

Embora a atuação do treinador de surf envolva elementos comuns aos de outras modalidades, as características do ambiente de prática e as indefinições conceituais do profissional que intervém nessa área suscitam desafios adicionais para a caracterização da prática pedagógica. Atento às propostas conceituais sobre a intervenção profissional no contexto esportivo e, mais recentemente, para os esportes de aventura, o objetivo deste estudo foi analisar as ações pedagógicas para o ensino do surf em relação às de planejamento, incluindo as ações durante e após a intervenção pedagógica, e ainda, as ações para a avaliação da aprendizagem. Para a obtenção dos dados, recorreu-se ao entendimento de profissionais com reconhecida experiência no ensino do surf, os quais atuam como formadores em cursos de capacitação de treinadores de surf, vinculados às entidades especializadas no ensino da modalidade no Brasil.

\section{PROCEDIMENTOS METODOLÓGICOS}

Realizou-se um estudo de abordagem qualitativa, com ênfase na descrição e na interpretação das informações (DENZIN; LINCOLN, 2008). Os dados foram obtidos a partir do estudo de casos múltiplos, o qual se caracteriza pela possibilidade de análise sistematizada e organizada de dois ou mais casos investigados no espaço de um mesmo conjunto de objetivos (STAKE, 2006). No presente estudo, participaram cinco formadores de treinador de surf (3 masculinos e 2 femininos), sendo que cada formador ou sujeito foi investigado individualmente, devendo cumprir um propósito específico dentro do objetivo global da pesquisa.

Nesse tipo de abordagem é possível analisar e descrever cada caso de forma detalhada e profunda, e, em momento posterior, realizar um estudo comparativo entre os casos, sugerindo possíveis regularidades e algumas especificidades entre eles, possibilitando, assim, a compreensão de um determinado fenômeno social complexo a partir da realidade de mais de um indivíduo (YIN, 2014). No presente estudo, o propósito foi garantir a obtenção de informações sobre o ensino do surf a partir de profissionais com experiência e conhecimento na formação de treinadores.

Os sujeitos foram selecionados intencionalmente, através dos seguintes critérios: ser atuante na formação de outros treinadores e treinador da modalidade, conforme Saury e Durand (1998) e Côté et al. (1995). No texto são identificados como (F1, F2, F3, F4 e F5).

A média de idade dos sujeitos era de 41,4 anos (mín. 36/ máx. 45); experiência de prática do surf de 32,6 anos (mín. 26/ máx. 39); experiência de prática como treinador, 20 anos (mín. 13/ máx. 28); experiência de prática como formador, 10,2 anos (mín. 6/ máx. 15). Quanto à certificação profissional, dois (F1 e F5) eram graduados em Turismo e provisionados pelo Conselho Regional de Educação Física (CREF) para o ensino do surf, dois (F2 e F4) graduados em Educação Física; e um (F3) provisionado pelo CREF para o ensino do surf. Todos os formadores tinham certificação em cursos para o ensino do surf, homologados pela Confederação Brasileira de Surf(CBS), Surfing Australia(F2, F4 e F5), National Surfing Scholastic Association (F2), British of Surfing Association (F2) e International Surfing Association (F4), e também certificação em salvamento aquático, com reconhecimento institucional, nacional e internacional. 
Um roteiro de entrevista semiestruturada foi utilizado para a coleta de dados, o qual se configura como um guia para o pesquisador direcionar a sua interação com o sujeito (SPARKES; SMITH, 2014). Foram contemplados quatro tópicos gerais sobre as ações pedagógicas: de planejamento da intervenção; ações durante a intervenção; ações após a intervenção; ações para a avaliação da aprendizagem. Quanto às ações pedagógicas durante a intervenção, utilizaram-se as indicações de Siedentop (1998) para classificar as informações emitidas pelos formadores em três dimensões: instrução, gestão/organização e clima relacional.

Após o preenchimento do termo de consentimento dos formadores, as entrevistas foram realizadas no local de trabalho de cada um dos sujeitos, com duração média de 89,5 minutos (mín. 69 min./ máx. 110 min.). As informações foram captadas por meio de gravador digital e armazenadas em microcomputador institucional. A pesquisa foi aprovada por um comitê de ética em pesquisa de uma universidade pública brasileira (Parecer $n^{0}$ 718.148).

Após a transcrição literal das entrevistas, utilizou-se o programa QSR Nvivo 10 para auxiliar no processo de análise delas, realizado em duas etapas: primeiro, fez-se a análise de cada caso individualmente; depois, o cruzamento dos casos, conforme Yin (2014). A codificação das transcrições brutas em representações de conteúdo foi realizada por meio da identificação das unidades de significado em função das categorias de análise. Na fase de cruzamento, o propósito foi identificar e agrupar as informações de cada formador em consensos. Para isso, agruparam-se, em um só local, todas as unidades de significado pertencentes a uma determinada categoria.

A análise dos dados, portanto, permitiu a identificação de consenso a respeito do entendimento dos formadores, possibilitando a criação de subcategorias, a partir do agrupamento das unidades de significado, nas categorias gerais estabelecidas a priori.

Para garantir a validade e a confiabilidade das informações, adotaram-se os seguintes procedimentos (SPARKES; SMITH, 2014; CÔTÉ et al., 1993; CULVER; GILBERT; SPARKES, 2012): a) realização de uma entrevista-piloto com um treinador experiente de surf, com o objetivo de aumentar a confiança e as habilidades do pesquisador para conduzir as entrevistas e também avaliar o roteiro de entrevista e os equipamentos; b) realização das entrevistas no ambiente de trabalho dos formadores para estimular informações mais detalhadas; c) checagem pelos sujeitos para confirmar a veracidade das informações; d) revisão por dois investigadores com experiência em pesquisa qualitativa para analisar o mesmo conjunto de dados e expor suas interpretações. Não foram identificadas discrepâncias entre elas.

\section{RESULTADOS}

Os resultados são apresentados por meio de mapas conceituais que correspondem a um tipo de representação no formato de diagramas, indicando relações entre os conceitos. Os indicadores, posicionados nas extremidades, referem-se ao entendimento consensual dos formadores acerca das ações pedagógicas que o treinador de surf deve realizar para 0 planejamento da intervenção, como as ações durante e após a intervenção, e as ações para a avaliação da aprendizagem. 
Figura 1: Ações pedagógicas de planejamento.

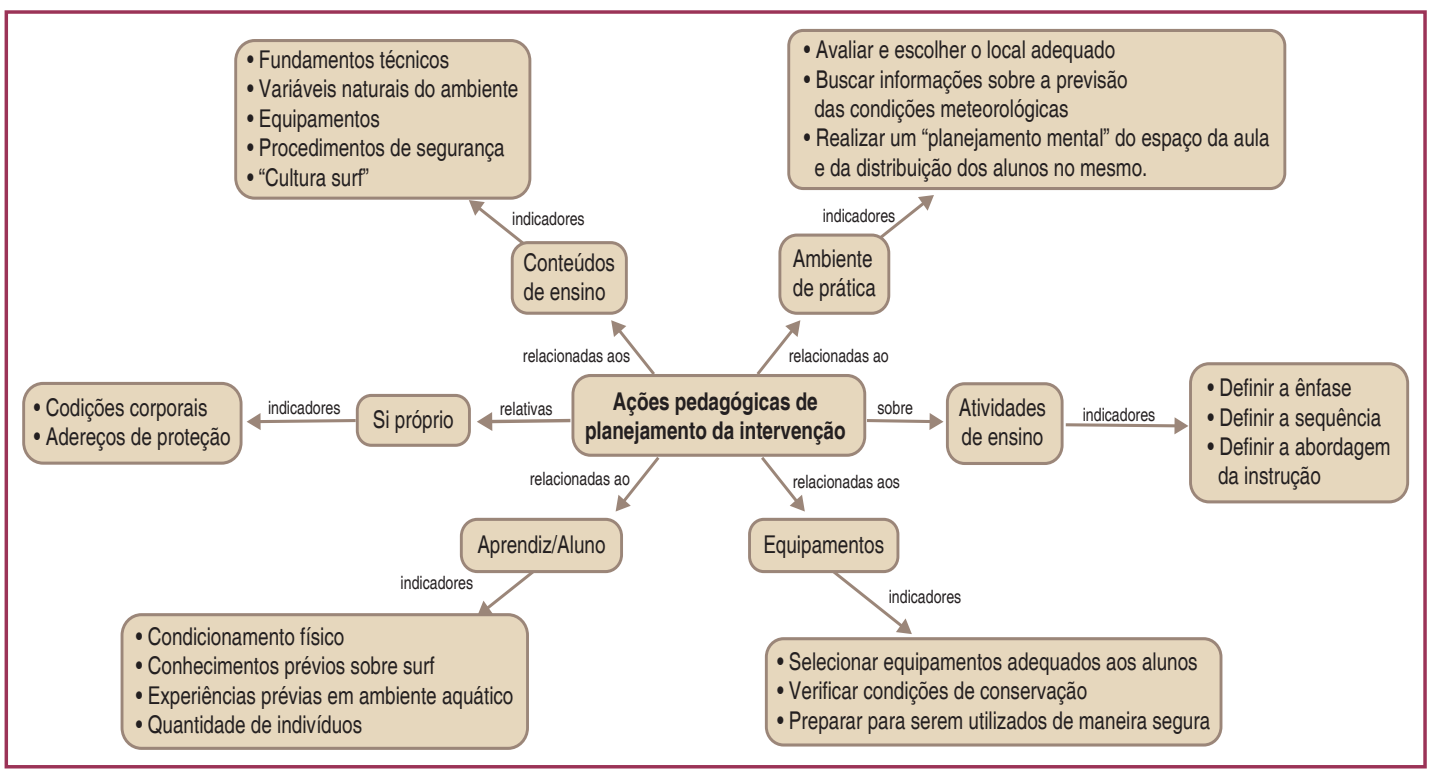

Fonte: dados da pesquisa.

Segundo os formadores, o planejamento corresponde à fase em que o treinador deve preparar sua intervenção, preponderando pensamentos de idealização e organização de suas ações pedagógicas, sejam elas referentes a uma única sessão/aula ou período, ou então, a uma temporada completa. Além de indicar aspectos comuns a qualquer modalidade esportiva (seleção dos objetivos, conteúdos e estratégias), os formadores mencionaram ações de planejamento relacionadas ao ambiente de prática do surf (praia/mar), aos equipamentos utilizados e aos aprendizes.

A respeito do ambiente de prática do surf, a fase de planejamento é fundamental para que o treinador possa definir "[...] qual vai ser o cenário [...]" (F1) da sessão, igualmente mencionado por F2:

0 treinador deve estar sempre observando o mar, observando o lugar onde vai trabalhar! Então, a preparação antes da aula, em primeira instância, é sobre o local que ele vai trabalhar... tem que dar aquela olhada geral para ver qual é o melhor ambiente para 0 aprendiz... o melhor lugar para ele ter mais segurança.

Para isso, o treinador deve estar atento "[...] às condições de variação de maré, direção de corrente, o formato do fundo... identificar um ambiente seguro" (F3), ou seja, realizar a interpretação do ambiente de prática que, na linguagem habitual do surf, é concebida como "leitura do mar". Essa leitura corresponde à compreensão que o treinador deve possuir acerca das condições do mar em um determinado instante, a fim de saber "[...] como essas variáveis estão influenciando ou vão influenciar a condição do mar nas próximas horas ou minutos" (F4).

As ações pedagógicas de planejamento acerca dos equipamentos devem abranger "[...] a escolha do equipamento adequado, ter a prancha em bom estado de conservação, os equipamentos de proteção individual, o leash', a roupa adequada [...]" (F3). Percebe-se que a escolha correta dos equipamentos, sobretudo a prancha, implicará o favorecimento de experiências positivas, ao minimizar riscos de desconforto, insegurança e fracasso durante a prática. De fato, o treinador "[...] tem que saber que a prancha, quanto mais área ela tiver, mais ela vai flutuar, quanto mais ela flutuar, mais fácil é para o aprendiz ficar de pé [...]" (F4).

Quanto às ações de planejamento acerca do aprendiz, os formadores mencionaram dois aspectos fundamentais: verificar as condições físicas e suas experiências prévias no esporte 
e/ou no surf. 0 primeiro diz respeito à identificação das características do praticante (físicas, cognitivas, afetivas, culturais...) para que o treinador possa elaborar o perfil ou a abordagem de sua intervenção, atendendo às necessidades e expectativas do aprendiz. Identificar as experiências prévias do praticante implica, diretamente, nos conteúdos que o treinador deverá contemplar, na profundidade que os tratará e nas estratégias que utilizará para atingir seus objetivos. Para o formador F1, o treinador deve "[...] descobrir realmente o que tem ali, naquele ser ali, para poder montar um treinamento que vá dar certo com ele [...]" (F1), e também no sentido de "[...] identificar situações que podem trazer um risco para ele mesmo [...]" (F4).

Figura 2: Ações pedagógicas durante a intervenção.

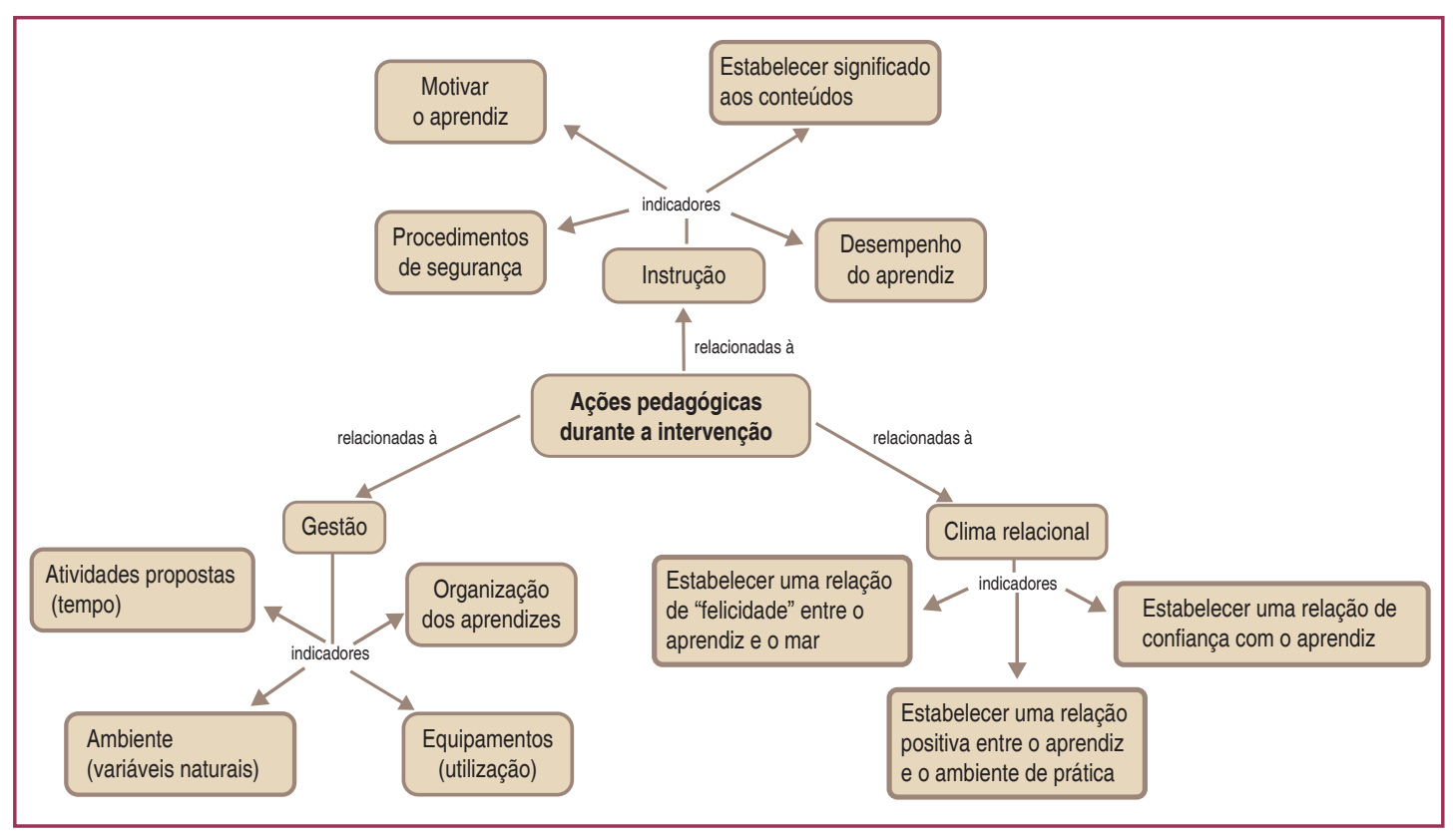

Fonte: dados da pesquisa.

As ações pedagógicas durante a intervenção correspondem àquelas situações em que 0 treinador interage com o aprendiz, estabelecendo as relações deste com o conteúdo, 0 ambiente e os demais aprendizes. Durante a prática do treinador, essas ações devem estar centradas em três dimensões: instrução, gestão/organização e clima relacional.

Os formadores destacaram a instrução verbal e a demonstração referentes aos comportamentos que o treinador deve emitir para fornecer informações acerca dos conteúdos e do desempenho esportivo do aprendiz, bem como elevar e manter a motivação e estabelecer uma comunicação clara e eficiente com o praticante. De acordo com F2, a instrução do treinador deve "[...] mostrar o que ele vai aprender, ajudar a escolher boas ondas, dar um feedback da onda que ele pegou, tentar corrigir os erros, ensinar o posicionamento deles [...]", além de "[...] motivar 0 aluno, principalmente, nos momentos que ele encontra dificuldade em realizar as atividades propostas, mantendo 'viva' a esperança dele alcançar o sucesso nas atividades". Além disso, os formadores salientam a importância da instrução do treinador para garantir a segurança do aprendiz, conforme afirma F5, ao dizer que: "[...] quando o treinador fala: 'vem aquil', é porque esse 'vem aqui' pode envolver algum risco para o aprendiz, então, é uma coisa que tem de deixar bem claro".

$\mathrm{Na}$ dimensão de gestão, as ações pedagógicas que o treinador deve adotar estão voltadas ao "controle" do espaço e dos recursos da sessão, sobretudo a disposição dos 
aprendizes dentro d'água, e também a gestão dos fatores que oferecem risco tanto aos praticantes quanto ao próprio treinador, conforme mencionam, respectivamente, F1 e F5:

[...] o treinador tem que estar conectado direto com o ambiente e o aprendiz. Estar atento com os banhistas, às vezes, ele está ali e aparece um banhista, eles ficam bem na linha da prancha [...].

[...] Então, o treinador tem que ser flexível, tem que saber se adaptar, faça chuva, faça sol, correnteza, tipos de fundos [...].

Outra dimensão das ações que o treinador deve realizar se refere ao estabelecimento de relações com os aprendizes, entre eles próprios, e entre eles e o ambiente de prática. Esse conjunto de relações sugere um tipo de sistema de interações, representado na citação de F1:

[...] a interatividade é muito importante! A aula não pode ser uma coisa mecânica. Não pode jamais! Tem que ser uma coisa que foi boa! Tem que ir estimulando, motivando, interagindo, senão fica monótono, fica chato, cria uma distância entre 0 treinador e 0 aluno... A gente tem que diminuir essa distância em relação ao treinador e ao próprio ambiente mesmo.

Conforme menciona F4, o treinador deve "[...] trabalhar com o aprendiz lá dentro da água numa fala apropriada, para tentar ficar amigo, tentar uma aproximação, para ele te dar as respostas e ficar à vontade dentro do mar [...]", o que, de fato, parece ir ao encontro do que F3 menciona a respeito de estabelecer uma relação de "felicidade":

Mesmo o mar sendo um ambiente inóspito, sendo um ambiente que exige resistência, sendo um ambiente que, aparentemente, pode ser perigoso... o treinador estabelecendo uma relação de felicidade, uma relação de amizade, uma relação de carinho com o aluno, vai ser possível, em $100 \%$ dos casos, que ele faça com que o aluno tenha uma relação de felicidade com o surf... com o mar e com a natureza.

Similarmente, para F5 "[...] o principal é o treinador conquistar a confiança do aprendiz. Ele, confiando no treinador, vai fazer o que o treinador pede e, com isso, o treinador vai ter mais respostas do aprendiz, e a coisa vai fluir melhor".

Figura 3: Ações pedagógicas após a intervenção.

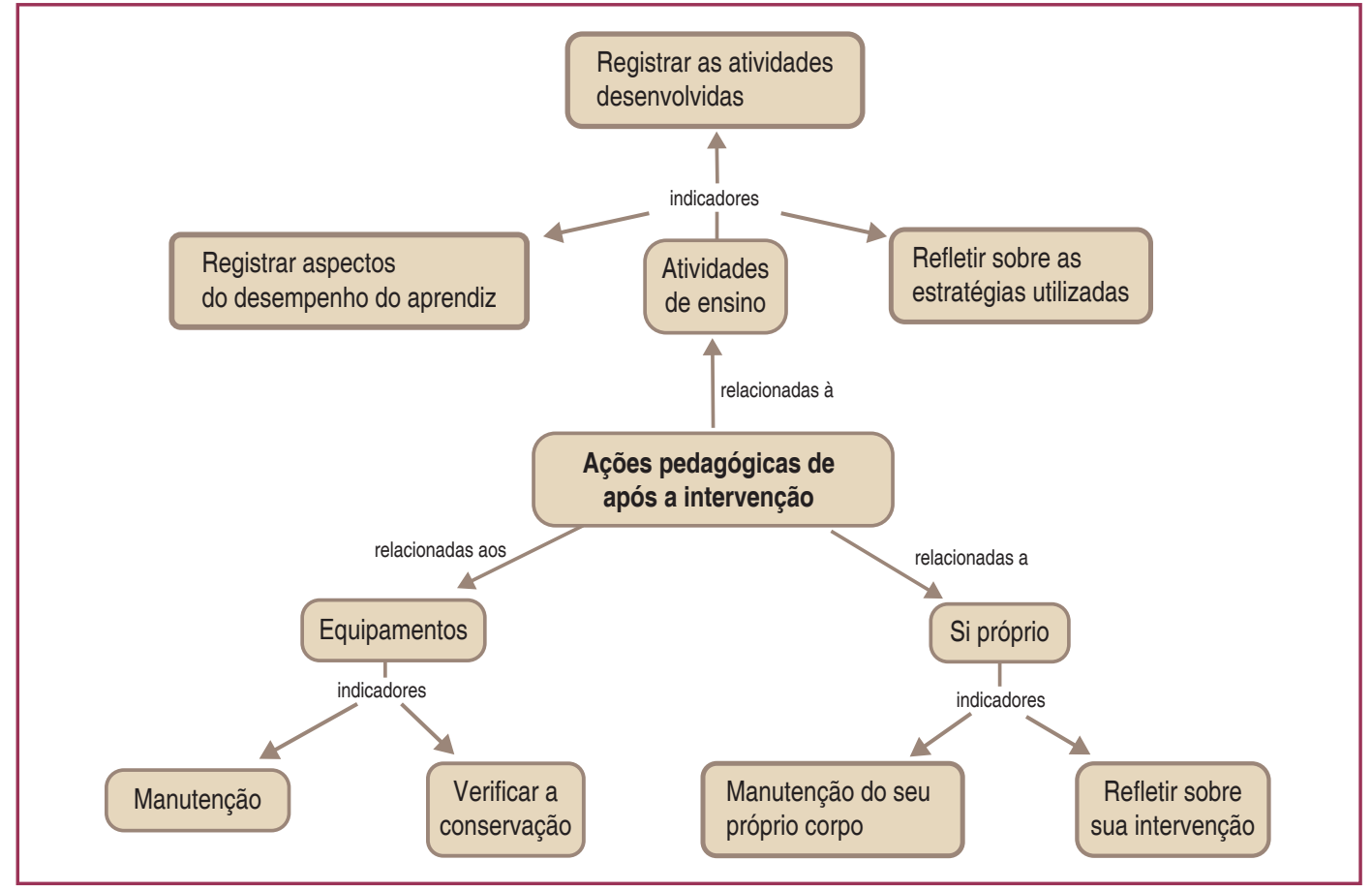

Fonte: dados da pesquisa. 
De acordo com os formadores, as ações pedagógicas do treinador, após a sua intervenção, devem se centrar em três aspectos: nas atividades de ensino realizadas em fase anterior, nos equipamentos utilizados e no próprio treinador.

Nessa fase, os formadores ressaltam a necessidade da elaboração de um registro das atividades desenvolvidas, conforme menciona F2: "[...] este é um momento que você tem para analisar o que aconteceu e, de repente, mudar para uma próxima sessão [...]". Para F4, 0 treinador deve elaborar "[...] um relatório de aula, pode ser escrito, é o ideal, então, colocar lá tudo que ele ensinou para o aluno, quais foram as dificuldades, quais foram os pontos que ele desenvolveu [...]", a fim de que o utilize para "[...] ele mesmo planejar a próxima aula e continuar a sequência pedagógica [...]" (F5). De fato, representa um momento favorável à reflexão sobre as estratégias utilizadas na fase anterior, de modo que o treinador possa identificar a necessidade de algum "ajuste" pedagógico e continuar progredindo no ensino.

Em relação aos equipamentos utilizados, as ações do treinador devem contemplar, prioritariamente, a sua adequada manutenção, e avaliar as condições de sua conservação para que a integridade dos aprendizes não seja colocada em risco. De acordo com F3:

Depois que 0 aprendiz vai embora, tem várias coisas que precisam ser realizadas. Número 1, é a correta manutenção do equipamento, não se deixa um minuto para depois... revisar a prancha para ver se houve alguma avaria, revisar o leash, guardar tudo de forma correta, para que possa ser utilizado de forma segura por outros alunos.

Relacionadas a si próprio, as ações do treinador devem estar voltadas, basicamente, para a manutenção de seu próprio corpo. Segundo os formadores, o ambiente de trabalho do treinador de surf (natural), e o modo como ele se envolve nas ações de ensino, ao assumir, geralmente, um papel ativo e exposto às diferentes variáveis naturais, gera uma demanda e um desgaste físico muito particular a essa atividade. Conforme menciona F1, "[...] às vezes, 0 treinador fica muito na água... é o dia inteiro molhado... então, tem que ter essa preocupação de estar se reidratando, se alimentando adequadamente [...]". Portanto, o treinador "[...] precisa se salvaguardar, se proteger, se hidratar, se alimentar bem [...]" (F3).

Figura 4: Ações pedagógicas para avaliar a aprendizagem dos praticantes.

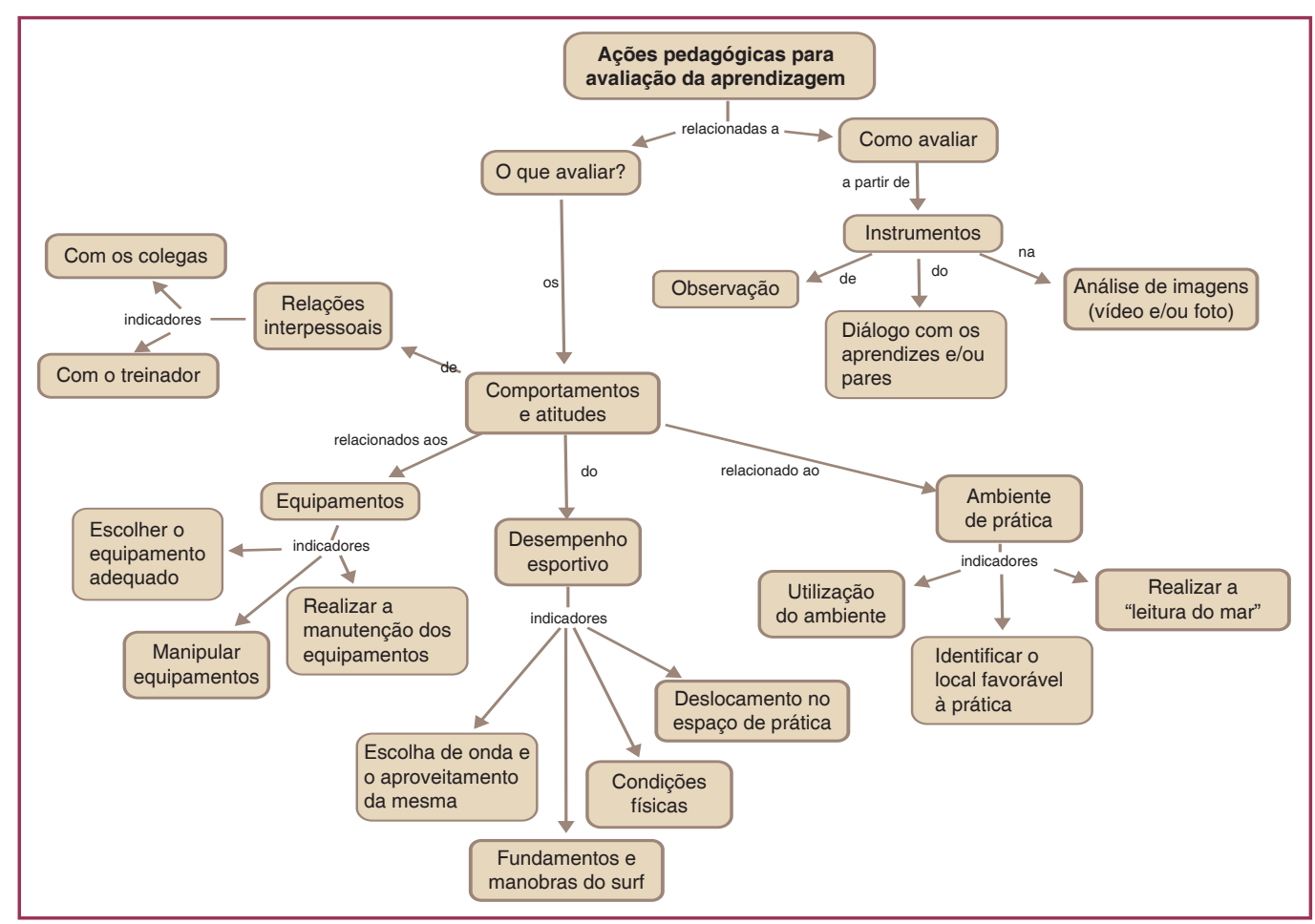

Fonte: dados da pesquisa. 
As ações pedagógicas do treinador para a avaliação da aprendizagem dos praticantes, segundo os formadores, podem ocorrer em diferentes momentos da prática do treinador (antes, durante e após), devendo considerar dois aspectos fundamentais: o que avaliar e os instrumentos a serem utilizados para avaliar.

A indicação dos formadores evidencia a preocupação sobre o que os treinadores devem avaliar no aprendiz, como, por exemplo, "[...] a execução do movimento. Se tu quer ensinar 0 aluno a ficar de pé, e vê que ele está de pé na prancha e indo embora, ele aprendeu [...]" (F4). Ou então, quando ele "[...] assume certa responsabilidade com o próximo, com 0 meio ambiente, um respeito, até na forma de como carrega a prancha, muda o comportamento [...]" (F5), são indicativos demonstrados por meio dos comportamentos do aluno ao manipular o equipamento, ao realizar os gestos do surf, ao selecionar o melhor equipamento para si, ao escolher o local para a prática e ao interagir com os colegas, o treinador e o mar, os quais podem representar alguma aprendizagem previamente planejada pelo treinador. Para tanto, segundo F2, o modo de avaliação mais viável para o treinador de surfé:

\begin{abstract}
A observação, com certeza! Você vai olhar, dar um feedback para o aluno, ele vai te responder na próxima onda. Você tem que observar para ver se ele entendeu 0 que você falou, às vezes, ele entendeu e não consegue realizar, mas em outras, ele entendeu e realiza, então, você tem certeza absoluta que ele entendeu e fez certo.
\end{abstract}

\title{
4 DISCUSSÃO
}

Considerando que as fontes desta pesquisa partem das declarações dos formadores sobre o ensino do surf, é relevante destacar que a discussão dos resultados apresentada a seguir centra-se no confronto entre as informações fornecidas pelos formadores e um conjunto de investigações que busca representar e conceituar as boas práticas dos treinadores de diversas modalidades esportivas, incluindo, recentemente, os esportes de aventura.

Os formadores investigados indicaram que as ações pedagógicas do treinador de surf envolvem planejar, realizar, instruir e gerir relações e recursos de aula, além de se comunicar, ensinar, avaliar, motivar e tomar decisões, ou seja, um conjunto de ações intencionais que visa produzir algum resultado específico no aprendiz (social, afetivo, psicológico, físico e esportivo), semelhante ao que a literatura especializada tem indicado (ABRAHAM; COLLINS; MARTINDALE, 2006; BARNSON, 2014; BENNIE; O'CONNOR, 2011; MESQUITA, 2000).

As ações pedagógicas, independentemente da modalidade, têm sido consideradas um elemento integrante da intervenção do treinador esportivo (ABRAHAM; COLLINS; MARTINDALE, 2006; BARNSON, 2014; BENNIE; O'CONNOR, 2011; CÔTÉ et al., 1995), geralmente vinculadas às crenças, aos conhecimentos e às características pessoais do próprio treinador e, também, ao contexto de intervenção, às características do aprendiz, entre outros. De acordo com Barnson (2014), trata-se de um elemento de ordem externa (comportamentos), mas que, inevitavelmente, está ligado aos atributos internos do treinador (trajetória pessoal, crenças, experiência e concepções).

Na proposta do Coaching Model (CM), elaborado por Côté et al. (1995), a partir do estudo com 17 treinadores de ginástica, verificou-se que as características pessoais do treinador e do atleta, assim como os fatores contextuais (denominados componentes periféricos), são 
utilizadas como referência para "idealizar suas ações". Similarmente, no Effective Coaching Model(ECM), elaborado a partir da percepção de 14 treinadores e 25 atletas de rúgbi e críquete, Bennie e O'connor (2011) verificaram que as ações para planejar, liderar, se comunicar e gerir pessoas estão diretamente relacionadas ao local de intervenção, às suas crenças e à cultura dos atletas.

Os resultados desse estudo indicaram que as ações do treinador de surf devem ser direcionadas, predominantemente, ao controle e ao ajuste das circunstâncias do ambiente de prática (mar, onda, ventos...), não só para atender às características e expectativas do praticante, mas também para a seleção e utilização de equipamentos adequados ao nível de conhecimento do aprendiz. De fato, a integração das informações a respeito desses elementos fornece um tipo de balizamento para as ações pedagógicas do treinador de surf, pois, ao mencionar o ambiente de prática do surf, ele precisa considerar todos os elementos que compõem e condicionam a onda do mar, a qual torna singular a prática dessa modalidade (RAMOS; BRASIL; GODA, 2013). Acredita-se, portanto, que a instabilidade do ambiente (natureza), típica dessa modalidade, pode levar o treinador a formular pensamentos e comportamentos distintos dos de treinadores que atuam em ambientes mais formais.

No estudo de Saury e Durand (1998) com cinco treinadores velejadores olímpicos, em ambiente similar ao do surf, as condições meteorológicas foram consideradas como um tipo de restrição às ações dos treinadores. No surf, essas restrições podem corresponder ao que Moreira (2009) denomina condicionantes da onda (direção e tamanho da ondulação, estrutura da costa, correntes, marés e ventos), as quais influenciam na altura, ângulo de deslocamento da arrebentação, intensidade e comprimento da onda. Ao entrevistar e observar a prática de 11 treinadores de surf portugueses (dois formadores), Correia (2005) verificou que todos se preocupavam em avaliar, constantemente, as condições de prática, processo este conhecido como leitura do mar e que implica na realização de situações de ensino adequadas aos aprendizes.

Segundo os formadores investigados no presente estudo, a leitura do mar e da onda, e a compreensão das condições do ambiente, de forma geral, possibilitam que o treinador realize adaptações importantes para a sua prática pedagógica. A interpretação do cenário implicará qualidade das decisões acerca da: escolha da praia com as melhores condições para a prática; seleção do local da praia, onde estão as melhores ondas para a prática; escolha da onda e do momento de auxiliar o aprendiz a deslizar na onda; possibilidade de realizar antecipações de situações inusitadas do ambiente. No estudo de Correia (2005), os treinadores de surf mencionaram duas hipóteses para a intervenção frente à instabilidade do ambiente: buscar um local adequado onde seja possível cumprir os objetivos da sessão, ou então, a adaptação do treino às condições apresentadas. Isso vai ao encontro da importância atribuída à interpretação do ambiente para a intervenção profissional em esportes de aventura, mencionada no Adventure Sports Coach Model, elaborado por Collins e Collins (2012; 2015).

As implicações da imprevisibilidade dos ambientes naturais (PAIXÃO et al., 2011) têm sido debatidas a partir do conceito de risco, configurando-se como um fator determinante na intervenção do treinador. Segundo Stranger (1999), no surf, o termo risco está associado às emoções e sensações (vertigem, adrenalina, autocontrole, comunhão, etc.) experimentadas durante a prática e, em menor proporção, à ameaça à integridade física do surfista, mais evidente em outros esportes de aventura. Portanto, de acordo com os formadores, a avaliação subjetiva 
que o treinador e o praticante devem realizar, a partir da relação com o mar e com a prática do surf, terá implicações tanto na prática do treinador como no nível de engajamento e satisfação do praticante na tarefa. Os níveis de risco percebidos pelo treinador e o praticante podem promover alterações no plano original da sessão do treinador, para atender aos condicionantes do ambiente de prática, ou, ainda, para aproveitar eventuais situações de facilitação de aprendizagem.

No estudo de Saury e Durand (1998) verificou-se que os treinadores adotam diferentes procedimentos ou modos de ação diante das restrições ambientais, a saber: rotinas para organizar a intervenção pedagógica (eficiência na gestão e condução do treino); antecipação mental das variações do ambiente a partir de planejamentos flexíveis (capacidade de prever eventos futuros, a reação dos participantes e as mudanças nas condições meteorológicas); a adaptação da intervenção aos imprevistos circunstanciais (definir especificidades em função das condições situacionais); a participação ativa do praticante nas decisões (relação treinadorpraticante para partilhar responsabilidades); e ainda, a utilização de experiências anteriores para poder intervir (optar por uma determinada ação que relembre experiências passadas).

Os procedimentos de "controle" da imprevisibilidade do ambiente, no âmbito dos esportes de aventura, têm sido tratados a partir do conceito de gestão do risco, correspondendo aos julgamentos, decisões e ações do treinador para garantir a segurança física do praticante e que facilite sua aprendizagem (COLLINS; COLLINS; GRECIC, 2014). Supõe-se, portanto, que o treinador de surf deva propor situações nas quais haja um nível de desafio que não exceda as capacidades de desempenho do praticante e, ao mesmo tempo, não seja mais baixo do que o nível de habilidade do indivíduo, pois, como indicam Collins e Collins (2013), o controle total dos riscos eliminaria a atratividade e as emoções proporcionadas aos praticantes.

Nessa perspectiva, acredita-se que o papel do treinador de surf é desenvolver habilidades e experiências que instrumentalizem o aprendiz a perceber e a gerenciar, com autonomia, o nível de risco mais ajustado às suas próprias expectativas. De acordo com Berry, Lomax e Hodgson (2015), isso demanda do treinador de esportes de aventura uma capacidade pessoal para se manter no ambiente de prática e uma profunda compreensão da natureza e do nível de risco favorável à aprendizagem, a fim de que possa se utilizar dos benefícios do risco como um modo de gerenciamento do próprio risco.

Segundo Collins e Collins (2013), a gestão de risco pode ser considerada um produto da experiência do treinador, que se manifesta em bons julgamentos das circunstâncias. Isso envolve a capacidade de reconhecer e integrar pistas sobre 0 ambiente, analisar opções e fatores situacionais e concretizar ações para garantir níveis adequados de risco. Ele deve idealizar um tipo de intervenção que envolva sua compreensão sobre os potenciais e as metas a alcançar com o aprendiz; depois, elaborar um modelo mental para atingir as metas, mobilizando e integrando diferentes tipos de conhecimentos; e, por fim, concluir o plano, por meio de suas ações pedagógicas típicas (CÔTÉ et al., 1995; GILBERT; TRUDEL, 2000).

No Authentic Coaching Model considera-se que os episódios da história pessoal do treinador servem como pontos de referência para suas decisões e ações futuras. Ao se depararem com uma situação de ensino real, os treinadores refletem sobre o evento em andamento, na tentativa de identificar se há similaridades entre a situação atual e outra já vivenciada. Ao constatar semelhanças entre ambas, utilizam a experiência vivida como referência para 0 presente, criando possibilidades para ações em um futuro imediato (BARNSON, 2014). 
Acredita-se, portanto, que as ações pedagógicas do treinador de surf são definidas a partir de um conjunto de restrições, particularmente, dos aspectos relacionados aos praticantes e da instabilidade do ambiente de prática do surf. Ao identificá-las, o treinador deve tomar decisões e agir para manter um ambiente de prática seguro e favorável à aprendizagem, conduzindo, gradativamente, o praticante para determinado nível de habilidade e entendimento sobre o surfe o ambiente.

Apesar de os modelos representarem conceitualmente práticas pedagógicas adequadas, deve-se considerar que a transferência direta dessas propostas para a intervenção do treinador está limitada ao caráter circunstancial e situacional que o contexto de prática exige. Neste sentido, os resultados de estudos empíricos, com esse propósito, podem ser utilizados apenas como princípios norteadores ou orientadores de práticas pedagógicas. No caso particular do surf, o dinamismo do ambiente natural exige do treinador uma elevada capacidade de reconhecer a singularidade das novas situações de ensino e de aprendizagem, com as quais ele se defronta no cotidiano de aula.

\section{CONCLUSÃO}

A prática pedagógica do treinador de surfocorre dentro do contexto onde se desenvolve a prática dessa modalidade: o mar. Deste modo, o propósito fundamental de suas ações é se ajustar ao dinamismo das ondas e suas condicionantes (vento, maré, ondulação...), bem como garantir níveis adequados de risco a fim de atender às necessidades e expectativas do aprendiz.

A concretização dessas ações decorre de um processo cognitivo que envolve crenças, concepções e experiências prévias do treinador, integradas a uma compreensão minuciosa do ambiente onde são aplicadas. Isso indica, de fato, que as ações do treinador têm ênfase no julgamento e na tomada de decisão, em detrimento de um predomínio do modo racional de pensamento.

A partir das entrevistas com os formadores, o presente estudo contribuiu para a construção de um roteiro conceitual útil à formação e à prática pedagógica de treinadores de surf. A intenção foi ampliar a sua base de conhecimento a fim de possibilitar a reflexão sobre situações específicas com as quais se deparam constantemente, bem como desenvolver habilidades para a resolução de situações dilemáticas de prática.

O estudo limitou-se a descrever e interpretar os casos particulares de cinco experientes formadores de treinadores de surf no contexto brasileiro. Para estudos futuros, sugere-se a realização dessas entrevistas com profissionais de diversas regiões do Brasil no intuito de ampliar os mapas conceituais construídos a partir do diálogo com os formadores e utilizar a observação sistemática da prática dos treinadores, de modo a complementar as informações obtidas por meio das entrevistas.

\section{REFERÊNCIAS}

ABRAHAM, Andy; COLLINS, Dave; MARTINDALE, Russel. The coaching schematic: validation through expert coach consensus. Journal of Sports Science, London, v. 24, n. 6, p. 549-564, 2006.

BARNSON, Steven. The authentic coaching model: a grounded theory of coaching. International 
BENNIE, Andrew; O'CONNOR, Donna. An effective coaching model: the perceptions and strategies of professional team sport coaches and players in Australia. International Journal of Sport and Health Science, Tokyo, v. 9, p. 98-104, 2011.

BERRY, Matt; LOMAX, Jane; HODGSON, Chris. Adventure sport coaching. London: Routledge, 2015. p. 357.

CASSIDY, Tania. Understanding the change process: valuing what it is that coaches do. International Journal of Sports Science and Coaching, Leeds, v. 5, n. 2, p. 187-191, 2010.

BLOOM, Gordon; SALMELA, John. Personal characteristics of expert team coaches. Journal of Sport Pedagogy, Plymouth, v. 6, n. 2, p. 56-76, 2000.

BOWES, Imornefe; JONES, Robyn. Working at the edge of chaos: understanding coaching as a complex, interpersonal system. The Sport Psychologist, Champaign, v. 20, p. 235-245, 2006.

CLARK, Christopher; PETERSON, Penelope. Teacher' thought processes. In: WITTROCK, Merlin. (Ed.) Handbook of Research on Teaching. 3.ed. New York: Macmillan, p. 255-296, 1986.

COLLINS, Loel; COLLINS, Dave. Conceptualizing the adventure-sports coach. Journal of Adventure Education Outdoor Learning, London, v. 12, n. 1, p. 81-93, 2012.

COLLINS, Loel; COLLINS, Dave. Conceptualizing the adventure sports coach. In: BERRY, Matt; LOMAX, Jane; HODGSON, Chris. Adventure sport coaching. London: Routledge, 2015. p. 5-23.

COLLINS, Loel; COLLINS, Dave. Decision making and risk management in adventure sports coaching. Quest, Illinois, v. 65, p. 72-82, 2013.

COLLINS, Loel; COLLINS, Dave; GRECIC, David. The epistemological chain in high-level adventure sports coaches. Journal of Adventure Education and Outdoor Learning, London, v. 15, n.2, p. 224238, 2014.

CORREIA, Marco Catarino Espada Estevão. Concepções didáticas e metodológicas do ensino do surf. 144 f. 2005. Dissertação (Mestrado) - Programa de Pós-graduação em Motricidade Humana, FMH, Lisboa, 2005.

CÔTÉ, Jean; GILBERT, Wade. An integrative definition of coaching effectiveness and expertise. International Journal of Sports Science Coaching, Leeds, v. 4, n. 3, p. 307-323, 2009.

CÔTÉ, Jean; SALMELA, John; BARIA, Abderrahim; RUSSELL, Storm J. Organizing and interpreting unstructured qualitative data. TheSport Psychologist, Champaign, v. 7, p. 127-137, 1993.

CÔTÉ, Jean; SALMELA, John; BARIA, Abderrahim; RUSSELL, Storm. The coaching model: a grounded assessment of expert gymnastic coaches' knowledge. Journal of Sport and Exercise Psychology, Champaign, v.17, p. 1-17, 1995.

CULVER Diane; GILBERT, Wade; SPARKES, Andrew. Qualitative research in sport psychology journals: the next decade 2000-2009 and beyond. The Sport Psychologist, Champaign, v. 26, p. 261-281, 2012.

CUSHION, Christopher; ARMOUR, Kathleen, JONES, Robyn. Locating the coaching process in practice: models 'for' and 'of' Coaching. Physical Education and Sport Pedagogy, Abingdon, v. 11, n. 1, p. 83-99, 2006.

DENZIN, Norman; LINCOLN, Yvonna. (Eds.). 0 planejamento da pesquisa qualitativa: teorias e abordagens. 2. ed. Porto Alegre: Artmed, 2008.

DIN, Cari; PASKEVICH, Dave. An integrated research model of Olympic podium performance. International Journal of Sports Science and Coaching, Leeds, v.8, n. 2, p. 431-444, 2013.

DUFFY, Patrick; HARTLEY, Hazel; BALES, John; CRESPO, Miguel; DICK, Frank; VARDHAN, Desirre; NORDMANN, Lutz; CURADO, José. Sport coaching as a "profession": challenges and future directions. International Journal of Coaching Science, Leeds, v. 5, n. 2, p. 93-123, 2011. 
DURAND-BUSH, Natalie; SALMELA, John. The development and maintenance of expert athletic performance: perceptions of world and Olympic champions. Journal of Applied Sport Psychology, Philadelphia, v. 14, p. 154-171, 2002.

GILBERT, Wade; RANGEON, Sandrine. Current directions in coaching research. Revista Iberoamericana de Psicología del Ejercicio y el Deporte, Las Palmas de Gran Canaria, v. 6, n. 2, p. 217-236, 2011.

GILBERT, Wade; TRUDEL, Pierre. Analysis of coaching science research published from 1970-2001. Research Quarterly for Exercise and Sport, Reston, v. 75, p. 388-399, 2004.

GILBERT, Wade; TRUDEL, Pierre. Validation of the Coaching Model (CM) in a team sport context. International Sports Journal, Leeds, v. 4, n. 2, p. 120-128, 2000.

JANUÁRIO, Carlos. Do pensamento do professor à sala de aula. Coimbra: Almedina, 1996.

JONES, Robyn; WALLACE, Mike. Another bad day at the training ground: coping with ambiguity in the coaching context. Sport, Education and Society, London, v. 10, n. 1, p. 119-134, 2005.

LYLE John. Sports coaching concepts: a framework for coaches' behaviour. Routledge: London, 2002.

LYLE, John. Modelling the complexity of the coaching process: a commentary. International Journal of Sports Science and Coaching, Leeds, v. 2, n. 4, p. 407-410, 2007.

MESQUITA, Isabel. A pedagogia do treino: a formação em jogos desportivos colectivos. 2. ed. Lisboa: Livros Horizonte, 2000.

MOREIRA, Miguel António de Almeida Garcia. Surf: da ciência à prática. Lisboa: FMH; 2009.

PAIXÃO, Jairo Antônio da; GABRIEL, Ronaldo Eugênio Calçada Dias; TUCHER, Guilherme; KOWALSKI, Marizabel; COSTA, Vera Lucia de Menezes. Risco e aventura no esporte na percepção do instrutor. Revista Psicologia e Sociedade, Florianópolis, v. 23, n. 2, p. 415-425, 2011.

RAMOS, Valmor; BRASIL, Vinicius Zeilmann; GODA, Ciro. O conhecimento pedagógico para o ensino do surf. Revista da Educação Física/UEM, Maringá, v. 24, n. 3, p. 381-392, 2013.

SAURY, Jacques; DURAND, Marc. Practical knowledge in expert coaches: on-site study of coaching in sailing. Research Quarterly for Exercise and Sport, Reston, v. 69, n. 3, p. 254-266, 1998.

SIEDENTOP, Daril. Aprender a enseñar la educación física. Barcelona: INDE, 1998.

SPARKES, Andrew; SMITH, Brett. Qualitative research methods in sport, exercise and health: from process to product. London: Routledge, 2014.

STAKE, Robert. Multiple case study analysis. New York: Guilford, 2006.

STRANGER, Mark. The aesthetics of risk: a study of surfing. International Review for the Sociology of Sport, London, v. 34, n. 3, p. 265-276, 1999.

YIN, Robert. Case study research: design and methods. Los Angeles: SAGE, 2014. 